\title{
EFFECTS OF LOADING DISTRIBUTION ON THE SEPARATED TRANSITIONAL FLOW OVER COMPRESSOR BLADES
}

\author{
Mingyang Wang \\ 1. Institute of Engineering \\ Thermophysics, \\ Chinese Academy of Sciences \\ 2. University of Chinese \\ Academy of Sciences \\ wangmingyang@iet.cn \\ Beijing, China \\ Shengfeng Zhao \\ 1. Institute of Engineering \\ Thermophysics, \\ Chinese Academy of Sciences \\ 2. University of Chinese \\ Academy of Sciences \\ zhaoshengfeng@iet.cn \\ Beijing, China
}

\author{
Ziliang Li \\ 1. Institute of Engineering \\ Thermophysics, \\ Chinese Academy of Sciences \\ 2.University of Chinese \\ Academy of Sciences \\ liziliang@iet.cn \\ Beijing, China \\ Yanfeng Zhang \\ 1. Institute of Engineering \\ Thermophysics, \\ Chinese Academy of Sciences \\ 2. University of Chinese \\ Academy of Sciences \\ zhangyf@iet.cn \\ Beijing, China
}

\author{
Chengwu Yang \\ 1. Institute of Engineering \\ Thermophysics, \\ Chinese Academy of Sciences \\ 2. University of Chinese \\ Academy of Sciences \\ yangchengwu@iet.cn \\ Beijing, China \\ Xingen Lu \\ 1. Institute of Engineering \\ Thermophysics, \\ Chinese Academy of Sciences \\ 2. University of Chinese \\ Academy of Sciences \\ xingenlu@hotmail.com \\ Beijing, China
}

\section{ABSTRACT}

The separated transitional flow on the suction surface of three compressor airfoils, characterized by equivalent diffusion level but different loading distributions, has been investigated through large eddy simulations (LESs) at a Reynolds number of $1.5 \times 10^{5}$. The baseline airfoil (V103-B) was redesigned to obtain an ultra-front-loaded (V103-UF) and an aft-loaded airfoil (V103-A). The turbulent statistics, vortex dynamics, and aerodynamic performance of the three airfoils were compared. A time-averaged laminar separation bubble (LSB) formed on the suction surface of V103-B and V103UF. Two-dimensional spanwise vortices shed periodically and were stretched with the amplification of Kelvin-Helmholtz (K-H) instability. Finally, three-dimensional hairpin vortices broke down into small turbulent eddies near the reattachment, along with the ejection-sweeping process of the near-wall flow. For V103-A, the turbulent separated layers failed to reattach on the blade surface. As such, the spanwise vortices shed randomly due to the strong interaction with the reverse flow. Furthermore, the perturbations in the separated shear layer propagated upstream and potentially triggered an absolute instability. By comparison, the LSB was suppressed on the suction surface of V103-UF. Therefore, the turbulent fluctuations in the separated flow were weakened, which contributed to significant profile loss reduction. Conversely, the performance of V103-A was degraded by the rapid turbulence generation in the extended reverse flow region.

\section{INTRODUCTION}

For the compression system in an aero-engine operating at high altitudes, the total pressure and temperature at the inlet decline to a low level. The Reynolds number $(\mathrm{Re})$ based on blade chord length may decrease by one order of magnitude. As such, the transition on the blade surface is delayed and the laminar flow region will be extended distinctly. Furthermore, the laminar boundary layer is prone to separate in the presence of strong adverse pressure gradient on the suction surface of compressor blades, which undergoes a transition process to turbulent via a laminar separation bubble (LSB) (Roberts, 1975). The laminar separation and the subsequent transition process will adversely affect the aerodynamic performance of compressors, including the adiabatic efficiency and the operating range (Balje, 1964; Wassell, 1968). Therefore, it is of great importance to accurately reveal the flow characteristics in the separated transitional flow and detect their underlying effects on the loss generation process.

In recent decades, numerous studies have been conducted to investigate the flow characteristics in the LSB and the associated effects on the aerodynamic performance of compressor cascades. Citavy and Norbury (1977) and Hobson et al. (1988) experimentally compared the profile loss of compressor cascades at various Reynolds numbers, they found that the performance degradation was attributed to the rapid growth of LSB. Numerical simulations were also conducted to add insight into the physical mechanisms 
responsible for the loss generation of compressor cascades. Medic et al. (2016), in a study of predicting the profile loss of compressor blades by large eddy simulations (LESs), proposed the transition process mainly determined the aerodynamic performance. Some researchers also focused on the loss mechanism in the transitional flow (Simoni et al., 2012), results showed that the roll-up and breakdown of largescale vortices were the decisive reasons for the rapid loss generation.

To control the transition process in the separated transitional flow and improve the aerodynamic performance of blades, several passive or active control approaches have been developed, such as dimples and V-grooves (Lake et al., 2000), rectangular bars (Volino, 2003), and pulsed vortex generator jets (Bons et al., 2001). The unsteady effects subjected to the upstream wakes were also considered to further increase the blade loading and reduce flow loss (Zhang and Hodson, 2005; Pacciani and Spano, 2006). Besides, an alternative flow control method was to modify the pressure gradient distribution (loading distribution) of airfoils, by which the velocity peak may move towards either the leading edge (front-loaded) or the trailing edge (aft-loaded). For example, some optimization methods have been applied to redesign the low-Re compressor airfoils, and the resulting performance variation before and after optimization was compared (Schreiber et al., 2004; Sonoda and Schreiber, 2007). Furthermore, the underlying mechanisms for the effects of loading distribution on the separated flow transition have also been investigated, it was supposed that the loading distribution determined the boundary layer state at the laminar separation, which consequently influenced the subsequent vortex evolution and the associated loss generation (Satta et al., 2014).

Although significant progress has been made towards a better understanding of the design strategy of low-Re airfoils and the boundary layer control methods, the detailed vortex dynamics in the separated transitional flow and the loss generation affected by loading distributions remain far from being completely understood. In the present study, a high subsonic compressor airfoil (V103-B) was redesigned to obtain two new airfoils with equivalent diffusion levels but different loading distributions (ultra-front-loaded: V103-UF and aft-loaded: V103-A). Large eddy simulations (LESs) were conducted to reveal the transient vortex structures and the instability mechanisms in the separated flow transition process at $\mathrm{Re}=1.5 \times 10^{5}$. Additionally, the underlying physics responsible for the loss generation of the three airfoils was analyzed and the aerodynamic performance was compared.

\section{GEOMETRIC MODEL AND NUMERICAL METHOD}

\subsection{V103 cascades}

The baseline airfoil used in the present study is a high subsonic compressor airfoil named V103-180 (V103-B), designed for the stator hub section in a highly-loaded compressor. The blade chord was extended to $180 \mathrm{~mm}$ to achieve a high resolution in measurements (Boese and Fottner, 2002). To investigate the effects of loading distribution on the separated transitional flow, the camber line and the thickness distribution of V103-B were modified to move the velocity peak towards the leading edge and the trailing edge, whereas the blade chord, stagger angle, and inlet /outlet flow angle remained unchanged. By which, an ultrafront-loaded (V103-UF) and an aft-loaded airfoil (V103-A) were obtained. The profiles of the three airfoils are presented in Figure 1, and the main geometric and aerodynamic parameters are listed in Table 1 .

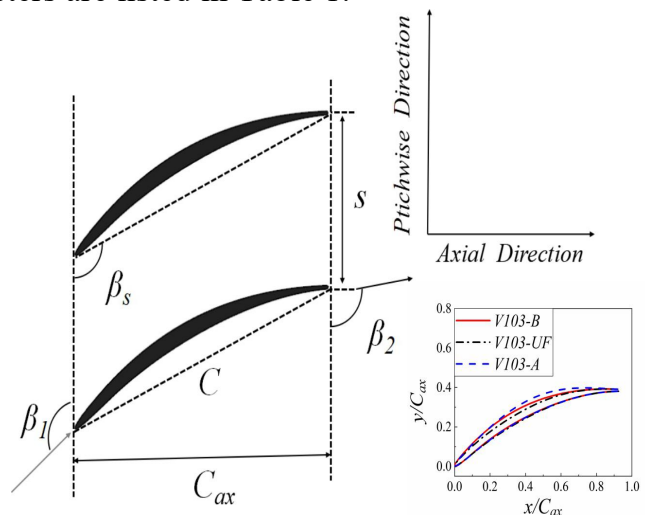

Figure 1 V103-B cascade

\section{Table1 Geometry and aerodynamic} parameters of $\mathrm{V} 103$ cascades

\begin{tabular}{ccc}
\hline Design parameter & Symbol & Magnitude \\
\hline Chord, mm & $C$ & 180 \\
Axial chord, mm & $C_{a x}$ & 166.3 \\
Pitch, mm & $S$ & 99 \\
Blade span, mm & $h$ & 27 \\
Inlet flow angle, ${ }^{\circ}$ & $\beta_{1}$ & 132 \\
Outlet flow angle, $^{\circ}$ & $\beta_{2}$ & 96 \\
Stagger angle, $^{\circ}$ & $\beta_{s}$ & 112.5 \\
Diffusion factor & $D$ & 0.42 \\
Inlet Mach number & $M a$ & 0.67 \\
Inlet Reynolds number & $R e$ & $1.5 \times 10^{5}$ \\
\hline
\end{tabular}

\subsection{Numerical methods and mesh}

The separated transitional flow characteristics of the present investigated cases were resolved by large eddy simulations (LESs), in which the time-dependent NavierStokes $(\mathrm{N}-\mathrm{S})$ equations are filtered in the physical space, and the large- and small-scale eddies are identified by comparing the mesh size and the scale of eddies. The so-called largescale eddies are solved directly while the effects of smallscale ones are modeled through the subgrid-scale (SGS) model. A wall-adapted local eddy-viscosity model (LES WALE model) (Nicoud and Ducros, 1999) was applied in the LES, one of the most significant advantages of this model is that almost no eddy-viscosity is produced in the wall-bounded laminar flows. Therefore, the laminar to turbulent transition can be revealed clearly. The spatial discretization was based on a central differencing scheme to avoid excessive dissipation, and a second-order backward Euler scheme was adopted for time marching.

The topology and resolution of the structured mesh were the same for all the cases, as shown in Figure 2. The 
normalized grid space in the streamwise, wall-normal, and spanwise directions satisfied the following restrictions: $\Delta x^{+}<20, \Delta y^{+}<1$, and $\Delta z^{+}<15$. The mesh resolution was supposed to be sufficient for the LES (Roberts and Yaras, 2006). The time-step was set as $1.5 \times 10^{-6} \mathrm{~s}$ to ensure the Courant-Friedrichs-Lewy (CFL) number was lower than 1 . Ten inner-loop iterations were required at each time-step before reaching the maximum convergence criteria of $10^{-4}$. Approximately 40 flow-through times (based on the axial chord and the axial velocity) were run to reach a statistical convergence state. Then, 8 flow-through times were calculated to obtain statistical data.

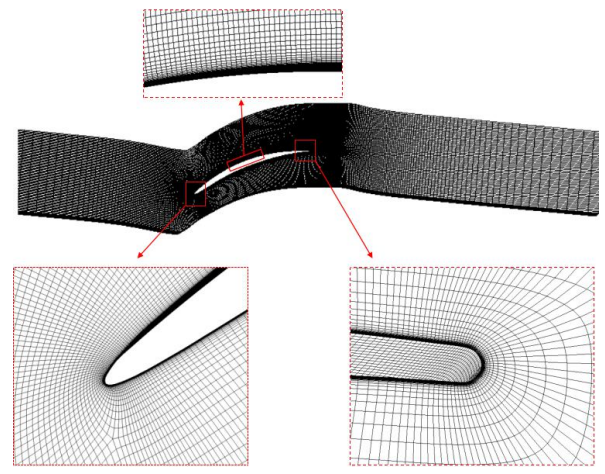

Figure 2 Computational mesh

\subsection{Boundary conditions}

To resolve the three-dimensional vortex structures in the transitional flow, the blade span was set as $0.15 \mathrm{C}\left(0.162 C_{a x}\right)$. The inlet plane was located at $150 \%$ of the axial chord upstream of the blade leading edge, and the total pressure $P_{01}$, total temperature $T_{01}$, and inlet flow angle $\beta_{1}$ were specified at the inlet boundary. The outlet plane was extended at $200 \%$ of the axial chord downstream of the blade trailing edge, and the average static pressure $P_{2}$ was given. Periodic conditions were imposed on the pitchwise boundary and the no-slip adiabatic conditions were applied on the blade surfaces.

\section{RESULTS AND DISCUSSION}

In the present study, the detailed vortex dynamics and the instability mechanisms in the separated flow over the suction surface of the three airfoils were analyzed at $\mathrm{Re}=1.5 \times 10^{5}$. Then the loss generation and the aerodynamic performance of the three airfoils were compared.

\subsection{Statistical flow characteristics of the three airfoils}

The predicted isentropic Mach number $\left(M a_{i s e n}\right)$ and the skin friction on the suction surface of the three airfoils were presented in Figures 3 and 4. For the baseline airfoil V103-B, the numerical result of $M a_{i s e n}$ was in close agreement with the experimental data (Boese and Fottner, 2002). The airflow on the suction surface continuously accelerated up to the velocity peak at $x / C_{a x}=20 \%$, then was diffused in the presence of strong adverse pressure gradient. The boundary layer detached from the blade surface at $x / C_{a x}=32.5 \%$, which underwent a transition process to turbulent and reattached at $x / C_{a x}=75 \%$. Correspondingly, a typical plateau (a nearly constant pressure region) was observed in the $M a_{i s e n}$ distribution, which indicated the laminar separation and subsequent transition process. Similarly, a plateau also appeared near $x / C_{a x}=20 \%$ on the pressure side. Therefore, a closed LSB formed on both the suction and pressure surfaces of V103-B. However, the present study only focused on the flow characteristics on the suction surface. For the ultrafronted airfoil V103-UF, a steep acceleration was observed very close to the leading edge. The laminar separation and turbulent reattachment occurred at $x / C_{a x}=34.5 \%$ and $x / C_{a x}=72 \%$, respectively. Therefore, the length of LSB was decreased by $5 \%$ of the axial chord compared with that of V103-B. However, for the aft-loaded airfoil V103-A, the flow acceleration near the leading edge was slower and achieved its velocity peak in the vicinity of $x / C_{a x}=30 \%$. As such, the laminar separation on the suction surface was delayed, which took place at $x / C_{a x}=39.5 \%$. Furthermore, although the skin friction was slightly positive in a short distance downstream of the separation point, the turbulent boundary layer appeared not to reattach at the rear part of the suction surface.

The time-averaged velocity contour and streamlines near the suction wall of the three airfoils were presented in Figure 5. A wedge-shape LSB formed on the suction surface of V103-B and V103-UF, and reverse flow was observed inside the LSB. By comparison, the length and the maximum thickness of LSB on the suction surface of V103-UF were smaller than those of V103-B. Therefore, ultra-front-loaded pressure distribution has the potential to suppress the development of LSB. For V103-A, however, the separated shear layer failed to reattach on the blade surface in the turbulent region. As a consequence, much reverse flow was accumulated near the trailing edge, causing serious flow blockage and wake mixing.

From the distribution of the root-mean-squared (RMS) velocity fluctuations shown in Figure 6 (the black dot line was the dividing line), the high-level velocity fluctuations were mainly concentrated at two regions demarcated by the diving line. The first region was very close to the suction wall in the aft part of LSB, which was attributed to the strong reverse flow mixing. Another region was located at the outer part of the separated shear layer, and the complicated vortex dynamics in the transition process was responsible for this. Due to the suppression of LSB on the suction surface of V103-UF, the highest level of velocity fluctuations was much smaller than that of V103-B, which contributed to significant profile loss reduction. Besides, a double-peak velocity fluctuation distribution was visible on the suction surface of V103-B and V103-UF, the outer peak was located along the dividing line, and the inner peak occurred at the reverse flow region. This phenomenon was also observed by McAuliffe and Yaras (2008). However, as the LSB burst on the suction surface of V103-A, the high-level velocity fluctuation region was extended distinctly, and as a consequence, the aerodynamic performance of the V103-A was severely deteriorated. 


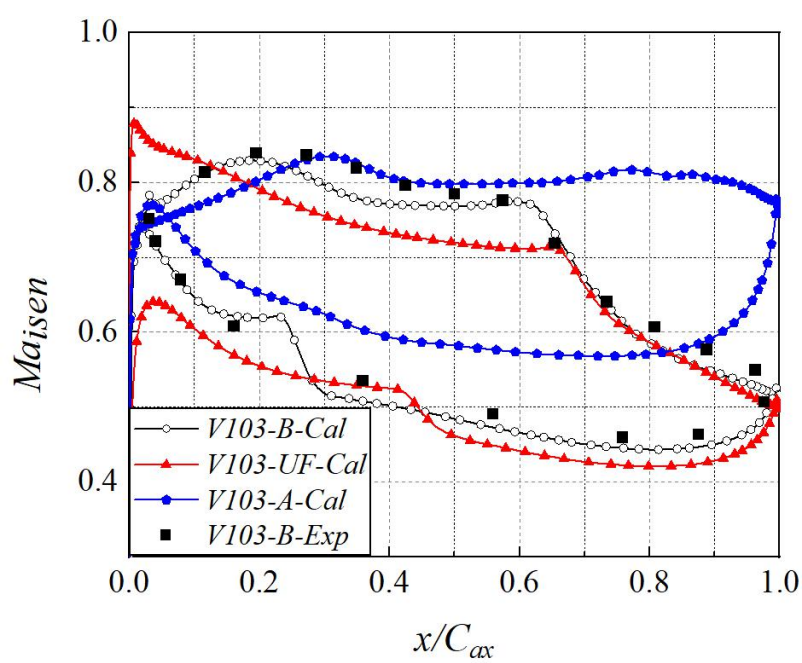

Figure 3 Isentropic Mach number of the three airfoils

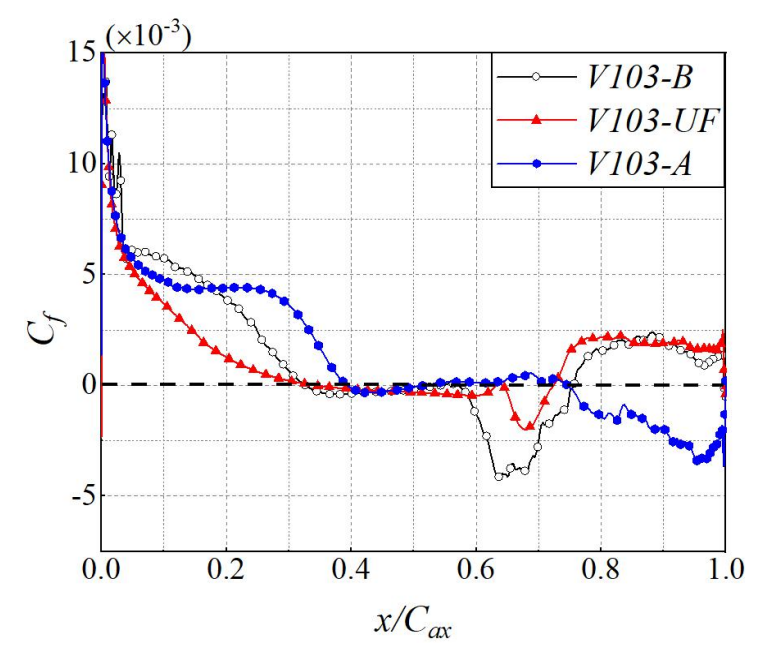

Figure 4 Skin friction on the suction surface of the three airfoils

\section{$U / U_{1}$

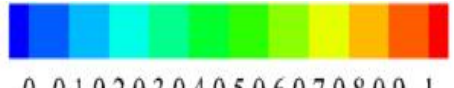

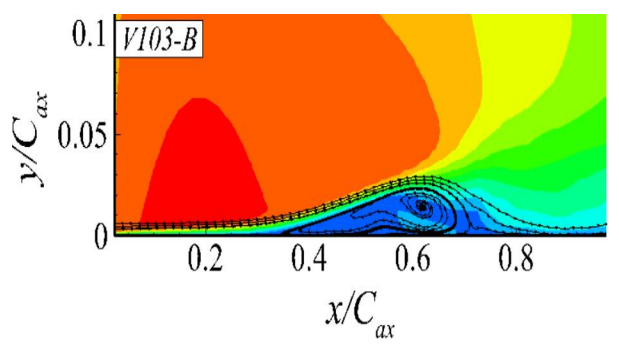
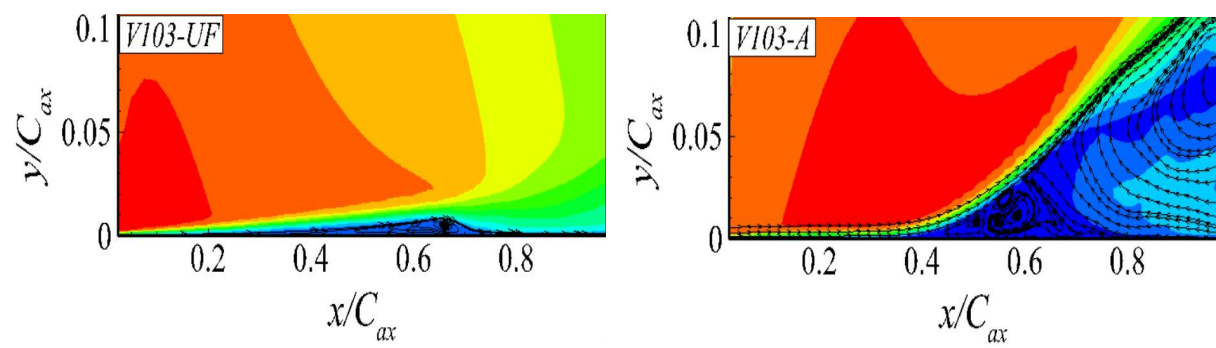

Figure 5 Time-averaged velocity on the suction surface of the three airfoils
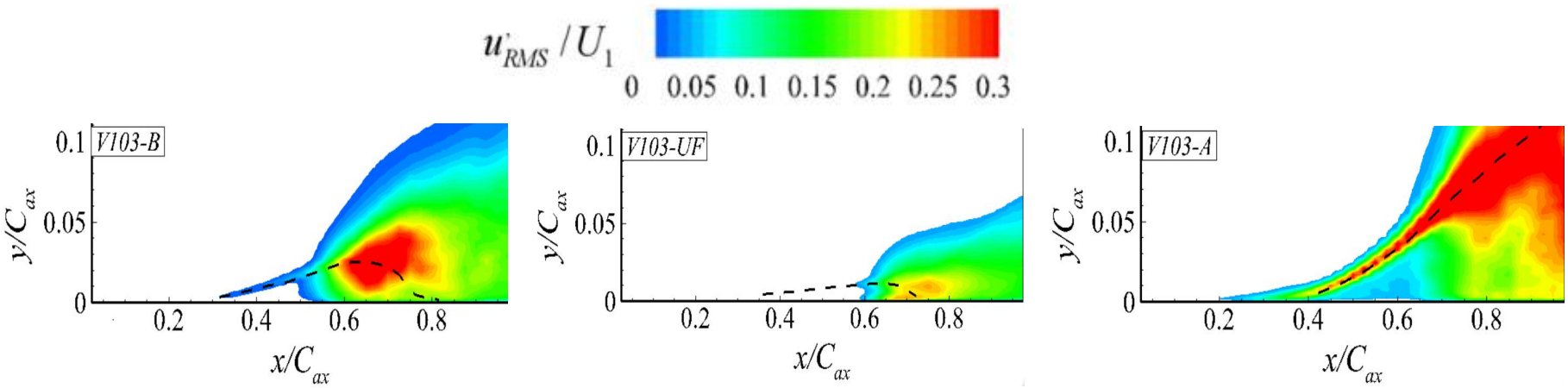

Figure 6 RMS velocity fluctuations on the suction surface of the three airfoils

3.2. Vortex dynamics on the suction surface of the three airfoils

The structures of spanwise vortices were identified by the Q-criterion (Dubief and Delcayre, 2000) and the isosurface was colored by the Mach number, as shown in Figure 7. For V103-B, two-dimensional spanwise vortices shed periodically from the upstream separated shear layer and were stretched towards the streamwise direction with the disturbance amplification. Then the strong interaction between spanwise and streamwise-evolving vortices in the vortex pairing process degraded their coherency and accelerated the distortion, leading to the local breakdown and small-scale turbulent eddies began to be generated. As time goes by, the highly distorted spanwise vortices evolved into hairpin vortices, which completely broke down into small turbulent eddies near the reattachment. Similar vortex evolution process was observed on the suction surface of V103-UF, the initial vortex shedding was also periodic and 
rolled up to form the vortex chains, but the strength of roll-up of the three-dimensional vortex chains was much weaker in the separated shear layer. For V103-A, due to the strong interaction with the reverse flow, the two-dimensional vortices shed irregularly and there was no specific frequency subjected to this. Moreover, the distortion rate of vortices seemed to be smaller than that of V103-B or V103-UF. Although hairpin vortices were also observable in the rear part of the suction surface, the vortex structures were more complicated.

The transient near-wall streamlines at the midspan were given in Figure 8. For V103-B and V103-UF, a clockwise vortex (main vortex) formed due to the flow instability in the separated shear layer, and multiple vortex cores merging and pairing inside the main vortex were also observed. In a short distance downstream of the main vortex, a counterclockwise vortex (secondary vortex) was induced as a consequence of the vortex pairing mechanism. As such, the near-wall flow from downstream was ejected reversely into the separated shear layer and was followed by a sweeping process, which enhanced the momentum exchange and forced the separated shear layer to reattach on the blade surface. Compared with the case of V103-B, the separated shear layer of V103-UF was thinner due to the suppression of LSB. Thus, the strength of reverse flow and the sweep-ejection process were much weaker. For V103-A, a large amount of reverse flow was accumulated in the turbulent separation region near the trailing edge. As the turbulent boundary layer failed to reattach, the ejection-sweeping process, which mainly determined the turbulent reattachment process, was not observed.

Additionally, it was worth addressing that the roll-up and breakdown of the large-scale vortices near the reattachment, along with the ejection-sweeping process of the near-wall flow, were mainly responsible for the rapid generation of turbulent fluctuations for V103-B and V103-UF. As the LSB was suppressed on the suction surface of V103-UF, the associated vortex dynamics were inhibited, and the turbulent fluctuations were consequently weakened. These results were in accordance with those observed in Figure 6. However, for V103-A, the strong reverse flow mixing at the rear part of the suction surface was supposed to dominate the generation of the highest-level turbulent fluctuations.

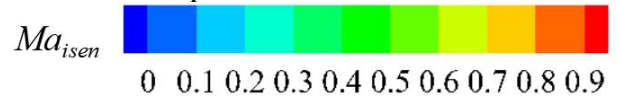

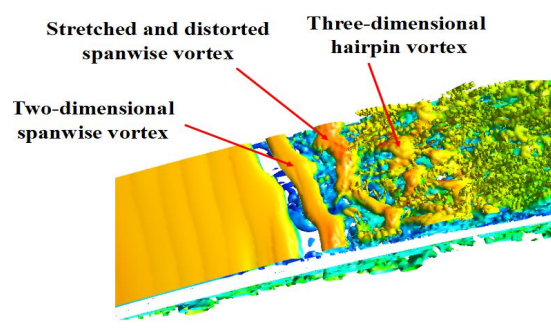

(a) V103-B

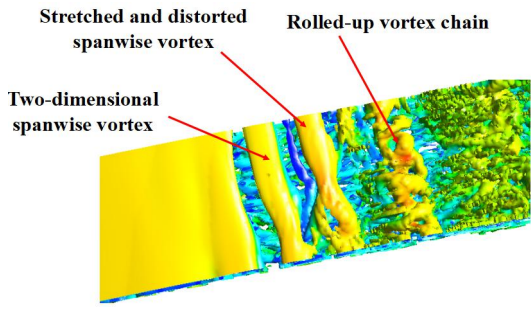

(b) V103-UF

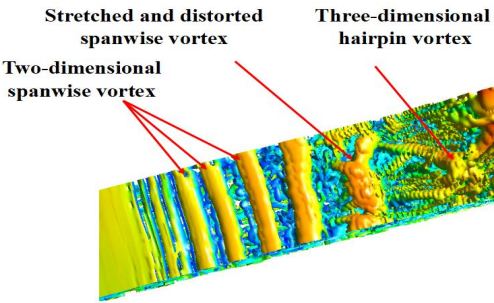

(c) V103-A

Figure 7 Transient spanwise vortex structures of the three airfoils: (a) V103-B, (b) V103-UF, and (c): V103-A
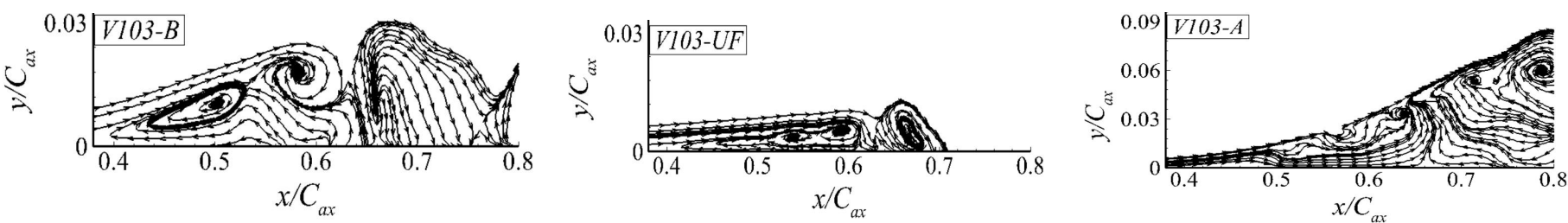

\section{Figure 8 Transient streamlines at the midspan}

\subsection{Transition mechanism in the separated flow on the suction surface of the three airfoils}

The transition process in the separated flow, closely interacted with the vortex dynamics and the instability amplification, was seriously affected by the loading distribution. Figure 9 compared the amplitudes of root-meansquared velocity fluctuations along axial direction for the three airfoils (the amplitudes have been transformed into a logarithmic form). For V103-B, exponential growth was observed between $x / C_{a x}=47.5 \%$ and $x / C_{a x}=57.5 \%$, which can be explained by the linear stability theory (LST). In the attached boundary layer, the initial growth of velocity fluctuations can be well predicted by the LST until a critical state was achieved, where the growth rate will deviate from the exponential path, indicating three-dimensional structures broke down and turbulent spots appeared. Similarly, the nonlinear amplification that appeared in the separated shear layer also corresponded with the three-dimensional breakdown and initiated transition (Lang et al., 2004; McAuliffe and Yaras, 2005). From Figure 9, the transition onset on the suction surface of V103-B occurred at $x / C_{a x}=57.5 \%$, which was related to the initial generation of small-scale turbulent eddies in the vortex evolution process 
(Figure 7). For V103-UF, although an adverse pressure gradient appeared very close to the leading edge, the diffusion was too weak to induce an earlier fluctuation amplification up to the location of $x / C_{a x}=50 \%$. Therefore, the transition onset on the suction surface of V103-UF was delayed, which occurred in the vicinity of $x / C_{a x}=65 \%$. It should be noted that the weaker adverse pressure gradient of V103-UF was also the decisive mechanism responsible for the LSB suppression. For the aft-loaded airfoil V103-A, the fluctuation amplitudes rapidly increased to a high level in the fore half part of the suction surface, indicating that the perturbations in the separated shear layer have propagated upstream. Then the fluctuations declined and were re-amplified in the presence of strong adverse pressure gradient at the rear part of the suction surface. It can be seen that the transition onset was delayed to $x / C_{a x}=75 \%$.

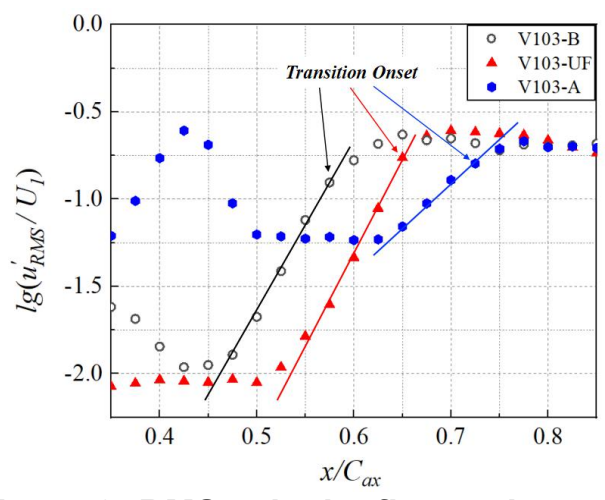

Figure 9: RMS velocity fluctuations along the axial direction for the three airfoils

To further add insight into the underlying physics dominating the transition process, the instantaneous pressure data at $x / C_{a x}=52.5 \%$ (in the separated shear layer) were stored to obtain the power spectrum density (PSD), as shown in Figure 10. For V103-B and V103-UF, a distinct peak was observed at the dominant frequency of $7731 \mathrm{~Hz}$, which was related to the periodic vortex shedding phenomenon (Figure 7). Due to the strong interaction with the reverse flow on the suction surface of V103-A, the two-dimensional spanwise vortices shed irregularly and there was no dominant frequency observed. The instability amplification in the separated transitional flow may be affected by the viscous TollmienSchlichting (T-S) or inviscid Kelvin-Helmholtz (K-H) mechanism, and the interaction between the two modes might also exist in some specific separated shear layers (Spalart and Strelets, 2000; Volino and Murawski, 2003; Roberts and Yaras, 2006). For the instability subjected to the K-H mechanism, the dominant frequency is often normalized by the boundary layer velocity and the momentum thickness at the laminar separation to create a Strouhal number $\left(S r_{\theta s}=f\right.$ $\left.\theta_{s} / U_{s}\right)$. The $\operatorname{Sr}_{\theta_{s}}$ numbers for V103-B and V103-UF cases were 0.00727 and 0.00849 , respectively, which were in close agreement with the typical ranges of K-H instability given in some published studies (Lin and Pauley, 1996; McAuliffe and Yaras, 2005). Therefore, it is believed that the inviscid $\mathrm{K}-\mathrm{H}$ mechanism governed the transition process on the suction surface of V103-B and V103-UF. However, due to the open separation on the suction surface of V103-A, the reverse flow was much stronger. As such, the viscous T-S instability within the near-wall region was enhanced and potentially triggered an absolute instability (Brinkerhoff and Yaras, 2011), which was demonstrated by the high RMS velocity fluctuations observed in the fore half part of the suction surface (Figure 9). Therefore, although the vortex rolling-up was observed in the separated shear layer, which indicated the existence of K-H instability, the important role of viscous instability should not be neglected in the transitional flow on the surface of V103-A, especially in the near-wall region.

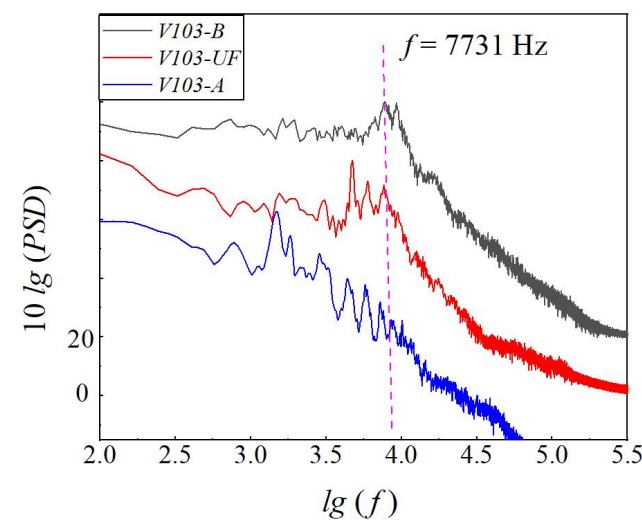

Figure 10: PSD comparison at $x / C_{a x}=57.5 \%$

\subsection{Aerodynamic performance comparison of the three airfoils}

As previously mentioned, the vortex dynamics in the separated flow mainly determined the production of turbulent fluctuations, which was supposed to be essential in the loss generation process (Simoni et al., 2012). The profile loss of the three airfoils at $\mathrm{Re}=1.5 \times 10^{5}$ was compared in Figure 11 . As the LSB on the suction surface of V103-UF was suppressed distinctly, the turbulent fluctuations in the separated flow declined to a low level. Therefore, the profile loss of V103-UF was decreased by $49.7 \%$ in contrast to that of V103-B. However, for V103-A, the strong adverse pressure gradient at the rear part of the suction surface failed turbulent reattachment, and a large amount of reverse flow was accumulated near the trailing edge. The increased turbulent fluctuation level caused by the reverse flow mixing enhanced the loss generation of V103-A. As a consequence, the profile loss of V103-A was increased by $202.8 \%$ and the aerodynamic performance was seriously deteriorated.

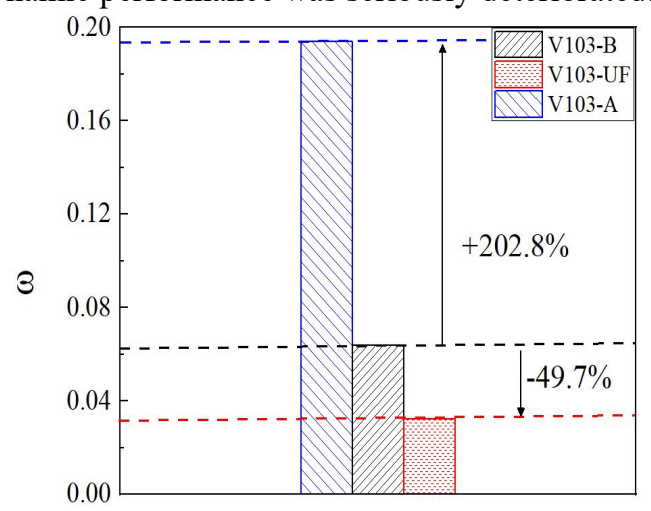

Figure 11: Profile loss of the three airfoils 
Figure 12 presented the boundary layer displacement thicknesses of the three airfoils, which can be used to directly compare the flow loss. In the attached boundary layer (before $x / C_{a x}=30 \%$ ), no distinct difference was observed, however, the boundary layer growth rate of V103-UF was much smaller than that of V103-B in the separated shear layer due to the LSB suppression. Conversely, the interaction with the strong reverse flow accelerated the boundary layer growth of V103A, resulting in more serious flow blockage and wake mixing loss near the trailing edge. From mentioned above, it can be concluded that the ultra-front loading distribution reduces the adverse pressure gradient and has the potential to effectively suppress the development of LSB, which weakens the turbulent fluctuation amplification and contributes to significant performance improvement.

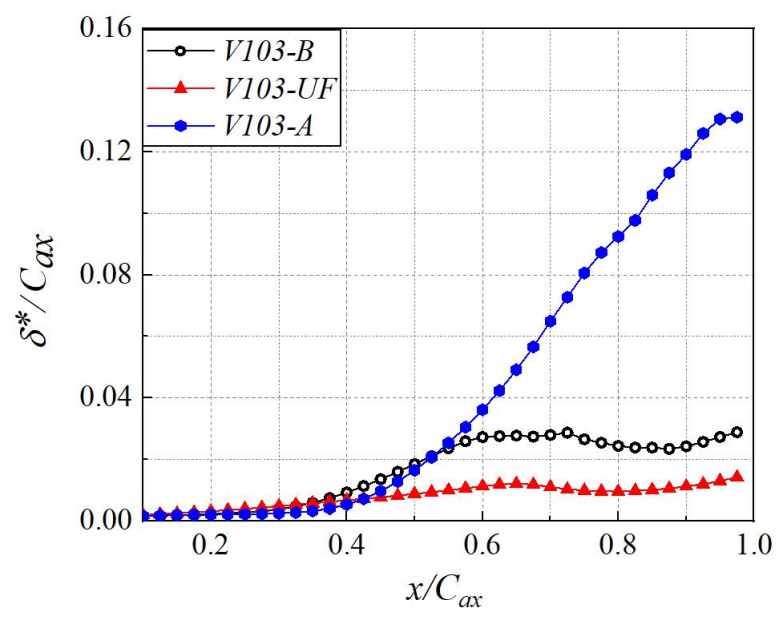

Figure 12: Boundary layer displacement thickness on the suction surfaces of the three airfoils

\section{CONCLUSIONS}

In the present study, large eddy simulations (LESs) were conducted to investigate the separated transitional flow over three compressor airfoils with different loading distributions (baseline: V103-B, ultra-front loaded: V103-UF, and aftloaded: V103-A) at a Reynolds number of $1.5 \times 10^{5}$, the vortex dynamics and the associated instability mechanisms governing the transition process were analyzed. Additionally, the loss generation mechanism and the aerodynamic performance of the three airfoils were compared. The main findings of this paper can be summarized as follows:

(1) A time-averaged laminar separation bubble (LSB) formed on the suction surface of V103-B and V103-UF. Compared with V103-B, the LSB on the suction surface of V103-UF was suppressed, and therefore, the turbulent fluctuations in the separated shear layer declined to a low level. For V103-A, the separated shear layer failed to reattach on the blade surface after the transition. As such, a large amount of reverse flow mixing at the rear part of the suction surface induced much stronger turbulent fluctuations.

(2) In the separated transitional flow, two-dimensional spanwise vortices on the suction surface of V103-B and
V103-UF shed periodically at the same frequency, then the spanwise vortices were distorted with the K-H instability amplification and evolved into three-dimensional hairpin vortices, which completely broke down into small turbulent eddies near the reattachment, along with the ejectionsweeping process of the near-wall flow. However, for V103A, the vortices shed randomly on the suction surface due to the strong interaction with the reverse flow. Moreover, the turbulent perturbations in the separated shear layer propagated upstream, indicating that absolute instability might be triggered.

(3) The strength of vortex roll-up and breakdown on the suction surface of V103-UF was suppressed due to the weaker adverse pressure gradient, which weakened the turbulent fluctuations and reduced the profile loss. However, as the separated shear layer failed to reattach on the suction surface of V103-A, the serious turbulent mixing in the reverse flow induced stronger turbulent fluctuations. Therefore, the aerodynamic performance of V103-A airfoil was rapidly deteriorated.

\section{NOMENCLATURE}

$\begin{array}{ll}R e & =\text { Reynolds number } \\ L S B & =\text { Laminar separation bubble } \\ P & =\text { Pressure } \\ T & =\text { Temperature } \\ M a & =\text { Mach number } \\ u_{R M S} & =\text { Root mean squared velocity fluctuations } \\ U & =\text { Velocity } \\ f & =\text { Frequency } \\ P S D & =\text { Power spectrum density } \\ \omega & =\text { Total pressure loss } \\ C_{f} & =\text { Skin friction coefficient } \\ \delta^{*} & =\text { Displacement thickness }\end{array}$

\section{SUBSCRIPTS}

$\begin{array}{ll}1 & =\text { At the inlet } \\ 2 & =\text { At the outlet } \\ 0 & =\text { Stagnation conditions } \\ a x & =\text { Axial direction } \\ \text { isen } & =\text { Isentropic conditions }\end{array}$

\section{ACKNOWLEDGMENTS}

The authors wish to acknowledge the financial support of the National Natural Science Foundation of China (Project No. 51836008) and the National Major Science and Technology Project of China (No.2017-II-0010-0024) for this project.

\section{References}

Balje, O.E. (1964). A Study on Reynolds Number Effects in Turbomachines. Journal of Engineering for Power, 86(3), pp. 227-235. doi:10.1115/1.3677584

Boese, M., and Fottner, L. (2002). Effects of Riblets on the Loss Behavior of a Highly Loaded Compressor Cascade. 
ASME Paper 2002-GT-30438. doi:10.1115/GT200230438

Bons, J. P., Sondergaard, R., and Rivir, R. B. (2001). Turbine Separation Control Using Pulsed Vortex Generator Jets. Journal of Turbomachinery, 123(2), pp.198-206. doi:10.1115/1.1350410

Brinkerhoff J R and Yaras M I. (2011). Interaction of Viscous and Inviscid Instability Modes in Separation-bubble Transition. Physics of Fluids, 23(12), pp.124102. doi: 10.1063/1.3666844

Citavy, J. and Norbury, J.F. (1977). The Effect of Reynolds Number and Turbulence Intensity on the Performance of a Compressor Cascade with Prescribed Velocity Distribution. Journal of Mechanical Engineering Science, 19(3), pp. 93-100. doi:10.1243/JMES_JOUR_1977_019_022_02

Dubief, Y., and Delcayre, F. (2000). On Coherent-Vortex Identification in Turbulence. Journal of turbulence, 1(1), pp. 011-011. doi:10.1088/1468-5248/1/1/011

Hobson, G.V, Hansen, D.J, Schnorenberg, D.G, et al. (1988). Effect of Reynolds Number on Separation Bubbles on Controlled-Diffusion Compressor Blades in Cascade. ASME Paper 88-GT-269. doi:10.1115/98-GT-422

Lake, J., King, P., and Rivir, R. (2000). Low Reynolds Number Loss Reduction on Turbine Blades with Dimples and V-grooves. AIAA Paper 00-0738. doi:10.2514/6.2000-738

Lang, M., Rist, U., and Wagner, S. (2004). Investigations on Controlled Transition Development in a Laminar Separation Bubble By Means of LDA and PIV. Experiments in Fluids, 36(1), pp. 43-52. doi:10.1007/s00348-003-0625-x

Lin, J. M., and Pauley, L. L. (1996). Low-Reynolds-number Separation on an Airfoil. AIAA Journal, 34(8), pp.15701577. doi: $10.2514 / 3.13273$

McAuliffe, B. R., and Yaras, M. I. (2005). SeparationBubble-Transition Measurements on a Low-Re Airfoil Using Particle Image Velocimetry. ASME Paper 2005GT-68663. doi:10.1115/GT2005-68663

McAuliffe, B. R., and Yaras, M. I. (2008). Numerical Study of Instability Mechanisms Leading to Transition in Separation Bubbles. Journal of turbomachinery, 130(2), pp. 021006. doi:10.1115/1.2750680

Medic, G., Zhang, V., Wang, G., Joo, J., and Sharma, O. P. (2016). Prediction of Transition and Losses in Compressor Cascades Using Large-Eddy Simulation. Journal of Turbomachinery,138(12), pp. 121001. doi:10.1115/1.4033514

Nicoud, F., and Ducros, F. (1999). Subgrid-scale Stress Modelling Based on the Square of the Velocity Gradient Tensor. Flow, Turbulence and Combustion, 62(3), pp. 183-200. doi:10.1023/A:1009995426001

Pacciani, R., and Spano, E. (2006). Numerical Investigation of the Effect of Roughness and Passing Wakes on LP Turbine Blades Performance. ASME Paper 2006-GT90221. doi:10.1115/GT2006-90221

Roberts, S. K., and Yaras, M. I. (2006). Large-eddy Simulation of Transition in a Separation Bubble. Journal of Fluids Engineering, 128(2), pp. 232-238. doi:10.1115/1.2170123

Roberts, W.B. (1975). The Effect of Reynolds Number and Laminar Separation on Axial Cascade Performance. Journal of Engineering for Power, 97(2), pp. 261-273. doi:10.1115/1.3445978

Satta, F., Simoni, D., Ubaldi, M., Zunino, P., and Bertini, F. (2014). Loading Distribution Effects on Separated Flow Transition of Ultra-High-Lift Turbine Blades. Journal of Propulsion and Power, 30(3), pp. 845-856. doi:10.2514/1.B34968

Schreiber, H. A., Steinert, W., Sonoda, T., and Arima, T. (2004). Advanced High-Turning Compressor Airfoils for Low Reynolds Number Condition - Part II: Experimental and Numerical Analysis. Journal of Turbomachinery, 126(4), pp. 482-492. doi:10.1115/1.1737781

Simoni, D., Ubaldi, M., and Zunino, P. (2012). Loss Production Mechanisms in a Laminar Separation Bubble. Flow, Turbulence and Combustion, 89(4), pp. 547-562. doi:10.1007/s10494-012-9407-y

Sonoda, T., and Schreiber, H. A. (2007). Aerodynamic Characteristics of Supercritical Outlet Guide Vanes at Low Reynolds Number Conditions. Journal of Turbomachinery, 129(4), pp. 694-704. doi:10.1115/1.2720868

Spalart, P. R., and Strelets, M. K. (2000). Mechanisms of Transition and Heat Transfer in a Separation Bubble. Journal of Fluid Mechanics, 403, pp. 329-349. doi:10.1017/S0022112099007077

Volino, R. J. (2003). Passive Flow Control on Low-Pressure Turbine Airfoils. Journal of Turbomachinery, 125(4), pp. 754-764. doi:10.1115/1.1626685

Volino, R. J., and Murawski, C. G. (2003). Separated Flow Transition in a Low Pressure Turbine Cascade: Mean Flow and Turbulence Spectra. ASME Paper, 2003-GT38727. doi:10.1115/GT2003-38727

Wassell, A.B. (1968). Reynolds Number Effects in Axial Compressors. Journal of Engineering for Power, 90(2), pp. 149-156. doi:10.1115/1.3609154

Zhang, X. F., and Hodson, H. (2005). Combined Effects of Surface Trips and Unsteady Wakes on the Boundary Layer Development of an Ultra-High-Lift LP Turbine Blade. Journal of Turbomachinery, 127(3), pp. 479-488. doi:10.1115/1.1860571 